\title{
Hot fusion
}

A team from Harvard University has demonstrated that human embryonic stem cells have the capacity to reprogram an adult cell nucleus, leading to a new way of deriving using oocytes.

Thanks to a recently published procedure (Hwang et al., 2005), generating genetically tailored human embryonic stem cell lines is now possible with reasonable efficiency by transferring a somatic cell nucleus of the desired genetic background into an enucleated oocyte. This resets the clock of the somatic nucleus to its embryonic state by an unknown mechanism that scientists are eager to understand. The technology, however, is at the core of the embryonic stem cell debate because it involves the destruction of the blastocyst formed from the manipulated oocyte. In addition, reliance on human oocytes is itself a source of ethical and logistical concerns.

Kevin Eggan and Chad Cowan, at Harvard University, sought an approach that would bypass this major issue. If the cytoplasm of an oocyte can do the reprogramming job, they asked, what about cytoplasm from its next of kin, an embryonic stem cell?

To test this alternative, they mixed a previously derived embryonic stem cell line with a fibroblast line, each tagged with a drug-resistance marker, in the presence of polyethylene glycol to induce cell fusion, and followed with double drug selection to isolate rare hybrids of the two cell types (Cowan et al., 2005). These hybrids are tetraploids but present the phenotype and the two most coveted characteristics of embryonic stem cells: capacity for immortal growth and pluripotency. Genome-wide gene expression analysis confirmed that, at the level of transcription, the embryonic stem cell program can win out.

This procedure promises to be a powerful tool to study the molecular mechanisms of reprogramming adult cells to their embryonic state, a theme that Eggan is most interested in pursuing. In the short term, he sees the importance of optimizing the technique, and also developing it into a system in which cell fusion is more efficient and it is possible to monitor whether cells go toward an embryonic or a differentiated fate after cell fusion.

Of course, the question burning on many people's lips is whether this technique can replace nuclear transfer into oocytes. Amid the attention that the paper has generated in the popular press, Cowan and Eggan have been prompt to point out that nuclear transfer is still well ahead from a technical perspective. The major hurdle of the fusion approach will be removing from the tetraploid hybrid the genetic material that belongs to the acceptor embryonic cell-a technical challenge which Eggan's team will also try to tackle.

"What we were looking for was a better way to study reprogramming," explains Eggan, "but there was also a realization that if this worked, one could imagine that based on this firm biological foundation, it might be possible to use embryonic stem cells themselves, even without understanding it, as a means to reprogram adult cells". The groundwork has certainly been laid now, and the prospect of replacing oocytes by a renewable resource is generating a lot of enthusiasm in the field.

\section{Veronique Kiermer}

\section{RESEARCH PAPERS}

Hwang, W.S. et al. Patient-specific embryonic stem cells derived from human SCNT blastocysts. Science 308, 1777-1783 (2005).

Cowan, C.A. et al. Nuclear reprogramming of somatic cells after fusion with human embryonic stem cells. Science 309, 1369-1373 (2005).

\section{CHEMICAL TOOLS}

\section{Precisely biotinylated RNA}

Using unnatural base pairs, researchers have site-specifically biotinylated RNA, allowing them to immobilize an aptamer on a chip and detect its interaction with a target protein.

Experiments with RNA often require immobilization of RNA molecules, which is usually achieved by labeling with biotin for immobilization on avidin supports. Using current techniques, one can incorporate biotinylated ribonucleotide monophosphates (for example, UMP or CMP) into RNAs, or only tag the RNA $5^{\prime}$ end by using a biotin derivative of the $5^{\prime}$ ribonucleotide monophosphate (for example, GMP for the conventional T7 promoter) in a transcription reaction. One can also chemically modify the $5^{\prime}$ or $3^{\prime}$ end of purified RNA. The simplest approach, of course, is to incorporate the label during transcription, but for some experiments it is important to site-specifically label the RNA at a location other than the $5^{\prime}$ end or to include only a single label to avoid altering the function of the RNA.

To achieve this, Ichiro Hirao and his colleagues at the University of Tokyo and at RIKEN modified unnatural base pairs such that a biotinylated base can be sitespecifically incorporated into RNA by T7 RNA polymerase. For example, 2-amino-6(2-thienyl)purine (s) can be incorporated into a DNA template. Then, in a standard transcription reaction, 2-oxo- $(1 H)$ pyridine (y) that had been biotinylated incorporates into the RNA transcript at sites complementary to s. This method can be easily adapted by any laboratory and can be used with commercially available transcription kits using T7 RNA polymerase. According to Hirao, "the available kits can be used without any changes of the protocol, except for using DNA templates containing unnatural bases such as $\mathbf{s}$ and substrates of $\mathbf{y}$ or modified $\mathbf{y}$."

In work described in a recent Nucleic Acids Research article, the group applied this method to biotinylate and immobilize an anti-Raf-1 RNA aptamer on sensor chips; this aptamer accurately recognized its target, the Ras binding domain of Raf-1, which was fused to a glutathione $S$-transferase reporter.

Hirao believes this unnatural base pair system will be very useful for RNA-based technologies. If the unnatural base pairs work with both prokaryotic and eukaryotic RNA polymerases, the system could be applied in a wider range of studies, including in vivo experiments. Hirao also plans to extend this system to function in replication, transcription and translation: "If the DNA fragments containing the unnatural base pair can be amplified by PCR, the unnatural base pair system will be a much more powerful tool." Irene Kaganman

\section{RESEARCH PAPERS}

Moriyama, K. et al. Site-specific biotinylation of RNA molecules by transcription using unnatural base pairs. Nucleic Acids Res. 33, e129 (2005). 